\title{
Hypocalcemia and hypokalemia due to hyperventilation syndrome in spinal anesthesia -A case report-
}

\author{
Hyun Soo Moon, Soo Kyung Lee, Ji Hoon Chung, and Chi Bum In \\ Department of Anesthesiology and Pain Medicine, Hallym University College of Medicine, Anyang, Korea
}

Hyperventilation syndrome (HVS) often occurs under stressful conditions, and has been reported during or after anesthesia and operation. HVS, characterized by multiple somatic symptoms and electrolyte imbalances induced by inappropriate hyperventilation, should be managed as an emergency. We report a rare case of HVS during spinal anesthesia. The patient was a previously healthy 51-year-old female without psychogenic conditions. During spinal anesthesia for lower extremity surgery, the patient complained of nausea, headache, paresthesia in the upper extremities and perioral numbness. We found carpal spasm in both hands and flattening of $\mathrm{T}$ wave on electrocardiogram (ECG). Emergent arterial blood gas analysis (ABGA) revealed markedly decreased $\mathrm{PaCO}_{2}$, hypocalcemia and hypokalemia. We managed the patient with verbal sedation, electrolytes replacement therapy and closed mask inhalation. HVS subsided gradually. We conclude that monitoring for possible HVS during anesthesia is very important for patient safety. (Korean J Anesthesiol 2011; 61: 519-523)

Key Words: Hyperventilation, Hypocalcemia, Hypokalemia.

Hyperventilation syndrome (HVS) is defined as over-breathing in excess of the metabolic needs of the body, removing more carbon dioxide than is produced and consequently results in respiratory alkalosis and a wide range of somatic symptoms [1]. Persistent respiratory alkalosis can induce secondary hypocalcemia and hypokalemia that may cause cardiac arrhythmias, conduction abnormalities, and various somatic symptoms such as paresthesia, hyperreflexia, convulsive disorders, muscle spasm and tetany [2]. HVS can occur when people become emotionally unstable, or in a temporarily unstable mental state. This is a condition affecting children as well as adults, and it predominates in female [3]. In the operating room, HVS has been reported as a cause of hypocalcema secondary to the environment and anxiety. [4].

We report a case of hypocalcema and hypokalema secondary to HVS identified during spinal anesthesia after observing paresthesia, numbness and spasm in both hands, confirmed by arterial blood gas analysis and changes in electrocardiogram,

Received: January 13, 2011. Revised: 1st, March 14, 2011; 2nd, April 21, 2011; 3rd, April 28, 2011. Accepted: April 29, 2011.

Corresponding author: Hyun Soo Moon, M.D., Department of Anesthesiology and Pain Medicine, Hallym University College of Medicine, 896 Pyeongchon-dong, Dongan-gu, Anyang 431-070, Korea. Tel: 82-31-380-3945, Fax: 82-31-385-3244, E-mail: hysomoon@yahoo.co.kr (c) This is an open-access article distributed under the terms of the Creative Commons Attribution Non-Commercial License (http:// creativecommons.org/licenses/by-nc/3.0/), which permits unrestricted non-commercial use, distribution, and reproduction in any medium, provided the original work is properly cited. 
and review the relevant literature.

\section{Case Report}

A 51 -year-old, $55 \mathrm{~kg}, 155 \mathrm{~cm}$ tall female patient with halux valgus was admitted to undergo osteotomy and internal fixation. The patient had a history of hypertension but no surgical history. Pre-operative serum electrolyte evaluation showed mild hypokalemia (3.3 mmol/L) and electrocardiogram (ECG) showed sinus bradycardia and non-specific T wave change (Fig. 1). Other blood tests and chest radiograph were unremarkable. For the treatment of hypertension, the patient did not take diuretics that can cause hypokalemia preoperatively. We explained to the patient spinal anesthesia before the operation and did not prescribe any premedication. In the operation room, the patient was monitored with non-invasive automated blood pressure, ECG with ST segment analysis and pulse oximetry. Her initial vital signs were as follow: blood pressure 150/90 $\mathrm{mmHg}$, heart rate 60 per minute, respiratory rate 18 per minute, and peripheral oxygen saturation of $97 \%$. To prevent perioperative hypotension, we infused Ringer's lactate solution (6 ml/ $\mathrm{kg}$ ) for 15 minutes before spinal anesthesia. We approached between the L4/5 levels using a $25 \mathrm{G}$ Quinke needle via the midline approach in the lateral recumbent position. After dural puncture, $10.5 \mathrm{mg}$ of $0.5 \%$ hyperbaric bupivacaine was injected slowly over 10 seconds. Ten minutes after administering spinal anesthesia, sensory blockade was verified up to the T8 thoracic dermatome by the pinprick test.

During surgery and anesthesia, there were no notable changes in vital sign and we neither supply oxygen nor sedatives. Approximately 90 minutes into the operation, the patient suddenly complained of nausea, headache, tingling sensation around the mouth, paresthesia in both arms and stiffness in both hands. We started administering oxygen to the patient at $5 \mathrm{~L} /$ min via a nasal cannula and her vital signs, including blood pressure, oxygen saturation and respiratory rate were within the
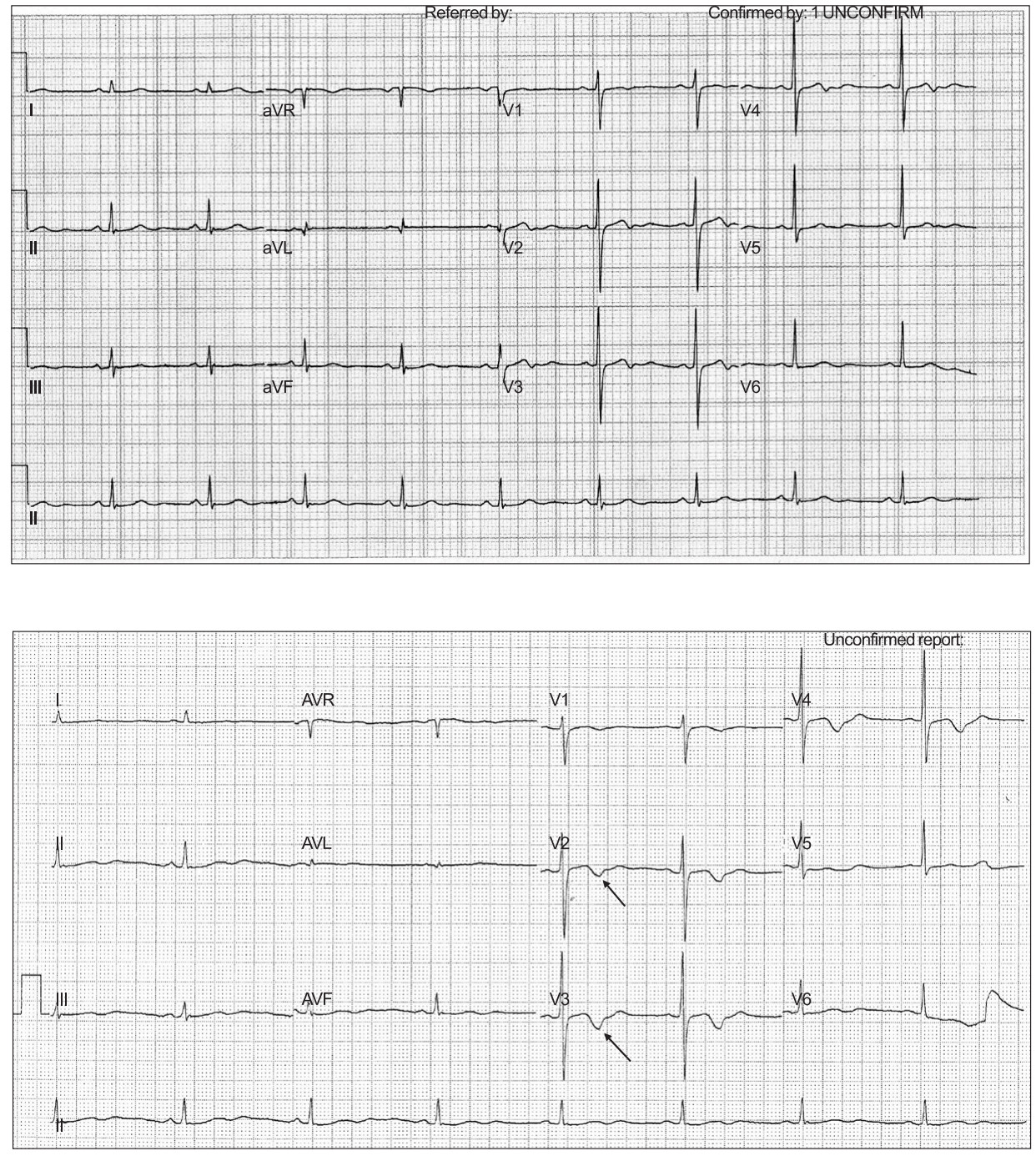

Fig. 1. Preoperative electrocardiogram shows sinus bradycardia and nonspecific $\mathrm{T}$ wave change.

Fig. 2. Intraopeative electrocardiogram shows second degree AV block, bradycardia and $\mathrm{T}$ wave inversion as indicated by arrows. 


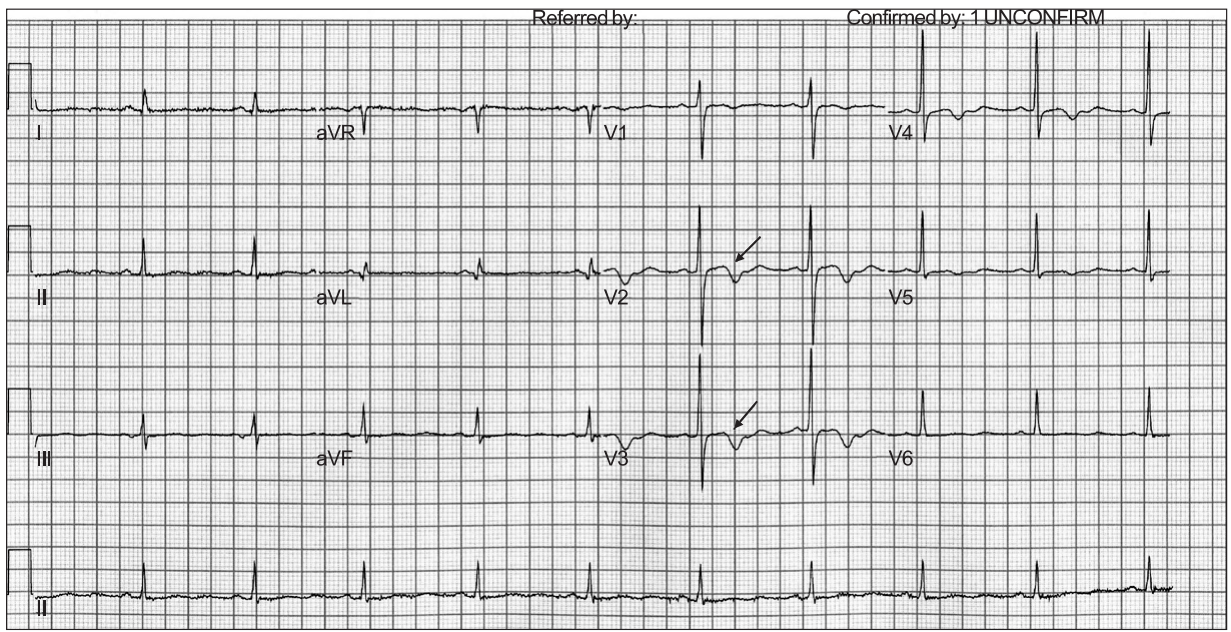

Fig. 3. Postoperative electrocardiogram shows normal sinus rhythm with partial recovery of $\mathrm{T}$ wave change, as indicated by an arrow. normal range, but she was bradycardic (48 per minutes). ECG showed second degree AV block and T wave inversion (Fig. 2). To determine the causes of the above symptoms and signs, we rechecked the anesthesia dermatome. The sensory blockade level slightly decreased to T10 but it was not considered as the main cause of her symptoms and signs. Emergent arterial blood gas analysis (ABGA) by radial artery puncture was performed, which showed normal $\mathrm{PaO}_{2}$ of $124 \mathrm{mmHg}$, but increased $\mathrm{pH}$ at 7.670, decreased $\mathrm{PaCO}_{2}$ at $26 \mathrm{mmHg}$, and increased plasma $\mathrm{HCO}_{3}$ - concentration of $30.0 \mathrm{mmol} / \mathrm{L}$. The patient was in a mixed state of severe respiratory alkalosis and mild metabolic acidosis.

HVS was assumed the main cause of respiratory alkalosis secondary to excessive spontaneous breathing during surgery and spinal anesthesia, as evidenced by severe hypokalemia (2.1 $\mathrm{mmol} / \mathrm{L})$, ionized hypocalcema $(0.72 \mathrm{mmol} / \mathrm{L})$ with somatic symptoms and signs of electrolyte imbalance. Verbal sedation and $4 \mathrm{mg}$ intravenous ondansetron were administered to alleviate nausea. Hyperventilation was controlled by closed mask ventilation with air/oxygen $(1.5 / 1.5 \mathrm{~L})$ per minute for one minute. In order to correct electrolye imbalance, which was the main cause of HVS symptoms and signs, $\mathrm{KCl} 10$ meq diluted in $50 \mathrm{ml}$ normal saline solution was administered over 30 minutes, and $10 \%$ calcium gluconate in $10 \mathrm{ml}$ was infused slowly over 5 minutes.

After the patient's hyperventilation subsided and at the time of completing intravenous electrolyte infusion, somatic symptoms, including perioral numbness, paresthesia of the both hands, fingers stiffness, nausea, and headache gradually improved. Repeat ABGA after returning the patient to the recovery room showed $\mathrm{pH} 7.48, \mathrm{PaCO}_{2} 48 \mathrm{mmHg}$ and $\mathrm{PaO}_{2} 72$ $\mathrm{mmHg}$, which was considerded improved alkalosis, increased potassium concentration of $3.0 \mathrm{mmol} / \mathrm{L}$, and slightly elevated ionized calcium of $0.96 \mathrm{mmol} / \mathrm{L}$. Second degree AV block on
ECG converted to sinus bradycardia and T wave inversion was also partially reversed.

After discussing with the orthopedic surgeon and a cardiologist, the patient was transported to a hospital room. The patient subsequently remained stable without symptoms and signs of HVS, and ECG on the ward (Fig. 3) reversed to preoperative baseline. The patient was discharged on the fifth postoperative day.

\section{Discussion}

HVS is a syndrome induced by excessive hyperventilation during anxiety, fear, and stress situations, which causes hypocapnia and the associated systemic symptoms and signs. A wide range of HVS symptoms are known to exist, and the pattern of symptoms differ by patient. During and after hyperventilation, tingling sensation in the hand and numbness in the feet, sweating, and symptoms of the central nervous system can be manifested [2]. The causes of HVS are classified as psychogenic, physiologic and organic. Anxiety and panic are thought to contribute to the cause of HVS but Garssen et al. [5] consider that the hyperventilation mechanism to be more complicated, because only some patients with panic disorder experience HVS. Air hunger with rapid breathing due to anxiety can also be the main cause of psychological HVS [6]. The list of respiratory and organic cause of hyperventilation includes asthma, chronic bronchitis, emphysema and chronic obstructive pulmonary disease (COPD) and rarely, pulmonary embolism, encephalitis, brain tumors and hypoparathyroidism.

In this case, the patient had no the history of COPD, no abnormal findings in pulmonary function test and history of pulmonary embolism which are usually associated with increased carbon dioxide levels, hypoxia and chest pain. As such, we did not think that HVS in this patient was due to 
organic cause. Physiologically, hyperventilaton can occur in women's menstral cycle. During the second half of the menstral cycle, increasing progesterone stimulates the central nervous system and cervical carotid body, which induce physiological hyperventilation [7]. Other physiological causes of HVS include long speeches or lectures, hypoxic conditions (for example in high altitudes), because hypoxia stimulates the respiratoy center to induce hyperventiltion. Therefore, in this case, environmental changes, such as anxiety and psychological trauma occur during anesthesia and surgery were regarded as the main causes of HVS. Before definitive symptoms and signs of HVS become evident, changes in breathing pattern should have been manifested. In this case, we did not recognize breathing pattern change visually by oversight, because the surgical site was in the feet and heated mattress covered the patient's trunk to prevent hypothermia. We suggest that in addition to vital sign monitoring, visual monitoring of the patient's breathing patterns and respiratory status during regional anesthesia are equally important to prevent HVS.

Respiratory alkalosis occurs when the partial pressure of carbon dioxide is less than $36 \mathrm{mmHg}$, which results in reduction in hydrogen $(\mathrm{H}+)$ ion in the intracellular fluid (ICF), and causes extracellular potassium ion shift into cells. As a result, serum potassium ion $(\mathrm{K}+)$ concentration decreases in order to maintain the balance of intracellular ions [8]. Serum $\mathrm{K}+$ concentration during respiratory alkalosis decreases in proportion to reduction in carbon dioxide partial pressure. Specifically, every $10 \mathrm{mmHg}$ decrease in $\mathrm{PaCO}_{2}$ causes concomitant reduction in serum $\mathrm{K}+$ by approximately 0.5 $\mathrm{mmol} / \mathrm{L}$ [9]. In this case, since the partial pressure of carbon dioxide had been reduced to $26 \mathrm{mmHg}$, calculation of the reduced blood $\mathrm{K}+$ concentration $(40-26 / 10=1.4) \times 0.5=$ $0.7 \mathrm{mmol} / \mathrm{L}$ is determined as a result. If the quantity of total potassium inside and outside the cells is already deficient, hypokalemic symptoms may intensify in alkalosis [10]. During anesthesia or surgery, administration of beta-stimulators, insulin or diuretics and hypothermia can induce hypokalemia [11]. However, in this case, there was no history of drug administration (beta agonists, diuretics) or hypothermia, and preoperative seum $\mathrm{K}+$ was mildly decreased to $3.3 \mathrm{mmol} / \mathrm{L}$. We assumed the patient's total potassium content was already low, which was thought a reason that caused HVS.

Therefore, preoperative mild hypokalemia without symptoms can rapidly deteriorate by spontaneous or iatrogenic hyperventilation during and after anesthesia. As such, adequate monitoring of $\mathrm{K}+$ concentration and expeditious correction are mandatory for patient safety, even in very mild hypokalemic state. ECG abnormalities associated with severe hypokalemia include $U$ waves greater than $1 \mathrm{~mm}$, $U$ waves larger than the $\mathrm{T}$ wave, $\mathrm{T}$ wave flattening or inversion (inverted $\mathrm{T}$ wave), and
QT waves [12]. ECG changes during hypokalemia usually do not match the severity of hypokalemia, and hypokalemia associated with other arrhythmogenic factors, such as magnesium deficiency, digitalis intoxication may induce severe cardiac arrhythymia, including atrial fibrillation and premature ventricular contraction.

In this case, besides second degree AV block and T wave inversion, moderarte bradycardia (48 rate/min) occurred during HVS. It was thought to be due to inhibition of sympathetic nervous system activity and reduction of venous drainage in spinal anesthesia in a patient who showed already preoperative bradycardia (60 rate/min). In this case, the heart rate did not increase despite HVS was caused by anxiety. We thought that the patient's antihypertensive therapy had prohibit heart rate increase but this remained unconfirmed. In addition, we did not consider administration of parasympathetic blockers because blood pressure and vital signs were normal. Instead, a decision was made to administer $\mathrm{K}+$ supplement to prevent cardiac arrhythmia progression. Whilst monitoring with ECG, we administered $10 \mathrm{mmol} \mathrm{K}+$ diluted in $50 \mathrm{ml}$ of normal saline over 30 minutes via peripheral vein, and we considered additional $\mathrm{K}+$ replacement after evaluated for post-infusion $\mathrm{K}+$ concentration (the standard method of intravenous $\mathrm{K}+$ replacement is to add $20 \mathrm{mmol}$ of $\mathrm{K}+$ to $100 \mathrm{ml}$ of normal saline and infuse this $\mathrm{K}+$ diluted solution over one hour) [13]. Our patient's symptoms and signs of HVS improved gradually, and we forfeited $\mathrm{K}+$ replacement.

Serum total calcium (normal: $8.0-10.2 \mathrm{mg} \%$, or $2.2-2.5$ $\mathrm{mmol} / \mathrm{L}$ ) is present in three forms, as follows: approximately $50 \%$ of the calcium is binded with the plasma protein(albumin is responsible for $80 \%$ ), unbounded ionized calcium, and 5-10\% calcium bounded and chelated with plasma anions, such as phosphoric acid, sulfuric acid, and citric acid. About half of total calcium is ionized calcium (normal: 4.0-4.6 $\mathrm{mg} \%$, or $1.0-1.5 \mathrm{mmol} / \mathrm{L}$ ) which is physiologically active. As such, hypocalcemia means ionized hypocalcemia clinically. Disorders commonly associated with ionized hypocalcemia include massive transfusion, hypoparathyroidism, renal failure, sepsis, acute pancreatitis, rhabdomyolysis, hypomagnesemia, and medication such as aminoglycosides, cimetidine, and heparin [14]. In this case, hypocalcemic symptoms manifested as perioral and bilateral hand numbness, limb stiffness, nausea, and headache towards the end of spinal anesthesia. Even though preoperative calcium, phosphorus and magnesium were not measured and preoperative hypomagnesia and hypocalcemia symptoms could be excluded clinically. HVS was thought to be the main cause of hypocalcemia as intraoperative ABGA showed severe respiratory alkalosis. Alkalosis promotes the binding of calcium to albumin and can reduce the fraction of ionized calcium in the blood, and ionized calcium may reduce 
without changes in total calcium. Hypocalcemic symptoms are more common with respiratory alkalosis than with metabolic alkalosis. The clinical manifestation of hypocalcemic symptoms are associated with overexcitabilty of sensory and motor neurons, which causes stiffness and spasms of the facial nerve, masticatory muscle spasm symptom (Chevostek's sign) and wrist spasm secondary to upper extremity ischemia during measurement of systolic blood pressure (Trosseau's sign). These hypocalcemic signs are neither specific nor sensitive and may not be manifested in hypocalcemia. Other symptoms of hypocalcemia may be manifested as cerebrovascular contraction headache and breathlessness [15]. Severe hypocalcemia (defined as ionized calcium $<0.65 \mathrm{mmol} / \mathrm{L}$ ) may be complicated with serious cardiovascular symptoms, such as ventricular tachycardia and refractory hypotension. Adequate calcium replacement therapy is crucial for patient safety during anesthesia and in the perioperative period.

In our patient, there was no wrist spasm during cuff inflation of non-invasive blood pressure intraoperatively, but wrist spasm occurred due to HVS at the end of surgery, and we confirmed HVS by decreased ionized calcium $(0.72 \mathrm{mmol} / \mathrm{L})$. Intravenous calcium supplementation includes $10 \%$ calcium chloride $(27 \mathrm{mg}$ calcium $/ \mathrm{ml}$ ) and $10 \%$ calcium gluconate (9.0-9.4 mg calcium/ $\mathrm{ml})$. Because the potency of both drugs are relatively large in $10 \mathrm{ml}$ and being hyperosmolar, and both forms of calcium are irritant and inflammatory to peripheral vessels, both agents need to be injected through the central vein via central venous catheter in order to prevent adverse effects. In this case, because the patient did not have central venous catheter, we chose and infused the less irritant calcium gluconate through the peripheral vein. In general, it is recommended that a bolus dose of 100$200 \mathrm{mg}$ of calcium diluted in $100 \mathrm{ml}$ isotonic saline, depending on the degree of hypocalcemia, should be infused over 10 minutes and continuous infusion of calcium $0.5-2 \mathrm{mg} / \mathrm{kg} / \mathrm{hr}$ for maintenance. Because the extent of hypocalcemia case was relatively mild in this case, we injected $10 \mathrm{ml}$ of $10 \%$ calcium gluconate (90 mg calcium) through the peripheral veins initially and resulted in increased ionized calcium $(0.96 \mathrm{mmol} / \mathrm{L})$. We did not administer additional calcium replacement treatment as her musculoskeletal symptoms showed gradual improvement.

Known respiratory intervention to manage rapid progressing hypocapnia in HVS includes the use of closed mask ventilation (rebreathing bag), but using performing closed mask ventilation in hypoxic patients or in patients with existing lung conditions may be dangerous, therefore special caution is required. Drug therapy for HVS includes the use of beta-blockers for patients with palpitations, sweating, or trembling but caution must be exercised in COPD patients at risk of brocho-obstructive disorders. To calm down anxiety or depression, sedative (benzodiazepine) use may be considered [7]. For regional anesthesia such as spinal anesthesia, we usually do not prescribe premedication and only when the patient or surgeon requests. If we had administered sedative premedication to our patient, it would have been useful to prevent HVS.

In conclusion, we diagnosed and managed HVS following ECG changes, and manifestation of symptoms and signs of hypocalcemia and hypokalemia induced by hyperventilation during spinal anesthesia. Anesthesiologists should always be aware of the possibility of electrolyte imbalance due to HVS during surgery or anesthesia, and in the perioperative period. In addition, timely and appropriate management for HVS is mandatory for patient safety.

\section{References}

1. Folgering H. The pathophysiology of hyperventilation syndrome. Monaldi Arch Chest Dis 1999; 54: 365-72.

2. Macefield G, Burke D. Paraesthesia and tetany induced by voluntary hyperventilation syndrome. Increased excitability of human cutaneous and motor axons. Brain 1991; 114: 527-40.

3. Sauty A, Prosper M. The hyperventilation syndrome. Rev Med Suisse 2008; 4: 2502-5.

4. Han MA, Ha KH. Carpal spasm due to hyperventilation syndrome after spinal anesthesia. Intravenous Anesthesia 2005; 9: 149-52.

5. Garssen B, Buikhuisen M, van Dyck R. Hyperventilation and panic attacks. Am J Psychiatry 1996; 153: 513-8.

6. Tobin MJ, Chadha TS, Jenouri G, Birch SJ, Gazeroglu HB, Sackner MA. Breathing patterns. 2. Diseased Subjects. Chest 1983; 84: 28694.

7. Gardner WN. The pathophysiology of hyperventilation disorders. Chest 1996; 109: 516-34.

8. Randall HT. Fluid, electrolyte, and acid-base balance. Surg Clin North Am 1976; 56: 1019-58.

9. Edwards R, Winnie AP, Ramamurthy S. Acute hypocapnic hypokalemia: an iatrogenic anesthetic complication. Anesth Analg 1977; 56: 786-92.

10. Freedman BI, Burkart JM. Endocrine crises. Hypokalemia. Crit Care Clin 1991; 7: 143-53.

11. Lipworth BJ, McDevitt DG, Struthers AD. Prior treatment with diuretic augments the hypokalemic and electrocardiographic effects of inhaled albuterol. Am J Med 1989; 86: 653-7.

12. Helfant RH. Hypokaemia and arrhythmias. Am J Med 1986; 80: 1322.

13. Kruse JA, Carlson RW. Rapid correction of hypokalemia using concentrated intravenous potassium chloride infusions. Arch Intern Med 1990; 150: 613-7.

14. Bushinsky DA, Monk RD. Electrolyte quintet: Calcium. Lancet 1998; 352: 306-11.

15. Hehrmann R. Hypocalcemic crisis. Hypoparathyroidism-nonparathyroid origin-the most frequent form: hyperventilation syndrome. Fortschr Med 1996; 114: 223-6. 